\title{
Efecto de microorganismos eficaces en los parámetros productivos y calidad de agua en el cultivo semi-intensivo de langostino blanco Litopenaeus vannamei.
}

Effect of effective microorganisms on production parameters and water quality in semi-intensive white shrimp Litopenaeus vannamei farming.

Dreime Toro M. ${ }^{1}$, Luis Llanco A. ${ }^{1}$, María Lora V. ${ }^{2}$, Rolando Tiparra T², Enrique Serrano-Martínez ${ }^{1}$.

\section{RESUMEN}

El objetivo de la presente investigación fue evaluar el efecto de microorganismos eficaces (EM) sobre el crecimiento y sobrevivencia de Litopenaeus vannamei (langostino blanco) cultivado en sistema semi-intensivo en el departamento de Tumbes, Perú. Para ello, post-larvas (PLs) de aproximadamente $0,02 \mathrm{~g}$ fueron sembradas $\left(15 \mathrm{PLs} / \mathrm{m}^{2}\right)$ en cuatro estanques (E) distribuidos en dos grupos: E1-E2 como controles (sin EM), y E3-E4 con suministro de EM. Se aplicó EM diariamente en el alimento y semanalmente en el agua de cultivo. De cada estanque, 200 langostinos fueron capturados semanalmente para evaluar el parámetro de crecimiento (peso). El análisis de varianza y la prueba de Tukey fueron usadas para determinar diferencias significativas en el crecimiento en peso. Los parámetros físico-químicos del agua fueron monitoreados (diariamente y semanalmente), por el contrario, la materia orgánica se determinó al inicio y término del cultivo. Finalmente se evaluaron los indicadores económicos del estudio. Se encontraron diferencias significativas del crecimiento en peso entre los grupos, pero no entre repeticiones, a favor del grupo suministrado con EM desde la quinta semana de cultivo. Se obtuvieron producciones brutas superiores: $1,001.28 \mathrm{~kg} / \mathrm{ha}$ (E3) y 1,028.90 kg/ha (E4), y un mejor Factor de Conversión Alimenticia (FCA): 1,11 00,31 (E3) y $1,23 \pm 0,29$ (E4) en el grupo EM. Se registraron sobrevivencias inferiores al 50\% en ambos grupos. Los parámetros físico-químicos del agua se mantuvieron dentro del rango óptimo para la especie estudiada. Los porcentajes de materia orgánica disminuyeron al término del cultivo en los estanques del grupo suministrado con EM; asimismo, los indicadores económicos fueron superiores en este grupo, destacándose el retorno por dólar invertido (EM=2,76; control:1,20). Podemos concluir que la aplicación de EM mejoró parámetros productivos y el FCA de L. vannamei, así como la calidad del agua, generando mayores ganancias económicas.

PALABRAS CLAVE: Acuicultura, Litopenaeus vannamei, probióticos, cultivo semi-intensivo

Facultad de Medicina Veterinaria y Zootecnia, Universidad Peruana Cayetano Heredia. Lima, Perú

Facultad de Ciencias Biológicas, Universidad Nacional Pedro Ruiz Gallo. Lambayeque, Perú 


\section{SUMMARY}

The objective of this study was to assess the effect of effective microorganisms (EM) on the growth and survival of Litopenaeus vannamei "white shrimp" reared in a semi-intensive system in the region of Tumbes, Peru. Post-larvae (PLs) of approximately $0.02 \mathrm{~g}$ were cultured $(15 \mathrm{PLs} / \mathrm{m} 2)$ in four ponds (E) distributed in two groups: E1-E2 as controls (without EM), and E3-E4 with EM supplementation. EM was added to feed daily and directly to the water weekly. From each pond, 200 shrimps were captured weekly to assess the growth parameter (weight). Analysis of variance and Tukey's test were used to determine significant differences in weight growth. The water physicalchemical parameters were monitored (daily and weekly), while, organic matter was determined at the beginning and at the end of the experiment. Finally, the economic indicators of the study were evaluated. Significant differences in weight growth were found between groups, in favor of the EM group since the fifth week of cultivation, but not in replicates. Higher gross productions were obtained: $1,001.28 \mathrm{~kg} / \mathrm{ha}$ (E3) and 1,028.90 kg/ha (E4), and a better Food Conversion Factor (FCA): 1.11-0.31 (E3) and 1.23-0.29 (E4) in the EM group. Survivals of less than 50\% were recorded in both groups. The physical-chemical parameters of the pond water remained within the optimal values for the studied species. Percentages of organic matter decreased at the end of the experiment in ponds of the EM group; economic indicators were also higher in this group, highlighting the return per dollar invested (EM: 2.76; control:1.20). In conclusion, the addition of EM improved parameters of production and FCA of L. vannamei, as well as water quality, generating better economic revenues.

KEY WORDS: Aquaculture, Litopenaeus vannamei, probiotics, semi-intensive culture.

\section{INTRODUCCIÓN}

La industria del langostino constituye uno de los sectores acuícolas que ha crecido a un ritmo más rápido a nivel mundial. En América, esta actividad se desarrolla desde el golfo de California hasta la parte norte del Perú, por lo general en zonas tropicales (Hendrickx, 1996; Jiang et al., 2000). En el Perú, el cultivo de Litopenaeus vannamei "langostino blanco" se realiza exclusivamente en las ciudades de Tumbes y Piura.

Actualmente el desarrollo de la actividad acuícola presenta numerosos retos tales como: incremento de los costos de producción, brotes de enfermedades e impacto de la contaminación ambiental. Una de las principales dificultades que existe en el rubro de la camaronicultura está relacionado con la deficiente asimilación del alimento suministrado, asociado con malas prácticas de cultivo (Paillard et al., 2004) y la calidad del agua (Pruzzo et al., 2005). Esto ha conllevado a un mayor interés en el empleo de productos eco amigables como por ejemplo bacterias probióticas que permitan mejorar, entre otros aspectos, el crecimiento de los organismos en cultivo, así como la calidad de agua y suelo (Nguyen et al., 2012).

Actualmente existe un número creciente de productos probióticos que permiten mejorar diferentes aspectos en la acuicultura, tales como la tecnología de los microorganismos eficaces (EM). Este probiótico, compuesto de bacterias ácido lácticas, nitrificantes y levaduras como Saccharomyces cerevisiae, incrementa la sobrevivencia y crecimiento de los organismos cultivados en sistemas controlados. Además, mejora la calidad del suelo y agua que es vertida a cuerpos acuáticos naturales al final del cultivo (Higa y Parr, 1994).

La evaluación de la efectividad de probióticos en el cultivo de langostinos y en la acuicultura en general ha sido abordada mayormente a nivel de laboratorio, abarcando líneas de investigación como la salud (Günther y Montealegre, 2004), nutrición (Chae et al., 2009) y calidad del agua (Campa et al., 2011). Por otro lado, estudios en campo en otros países productores de L. vannamei en sistemas semiintensivos, como Colombia y Ecuador, aplicando EM (Rhodopseudomonas palustris, Lactobacillus plantarum, L. casei, y S. cerevisiae), han reportado resultados de crecimiento por debajo de los esperado para un tiempo estándar de cultivo de 120 días (Villamil y Silva, 2009; Novillo, 2005; Guillén, 2005). Sin embargo, otras experiencias han demostrado que el uso de estos EM mejoró los parámetros de calidad del agua, en cuanto a niveles de oxígeno disuelto (Tejada et al., 2002), y redujo los ciclos de cultivo (Ortega y Encalada, 2003).

Dado que el uso de la tecnología EM en acuicultura sigue siendo controvertido en cuanto a su eficacia, al no haberse estandarizado su aplicación en campo 
(Farzanfar, 2006; Kesarcodi et al., 2008), es importante contrastar experimentalmente si su aplicación mejora el crecimiento y consecuentemente la producción de L. vannamei cultivados en un sistema semi-intensivo. Esto permitiría destacar la importancia económica de la implementación del probiótico en este rubro. El objetivo de esta investigación fue determinar y comparar el crecimiento y supervivencia de $L$. vannamei suplementado con EM, así como realizar una evaluación económica del suplemento.

\section{MATERIAL Y MÉTODOS}

\section{Lugar de estudio y diseño experimental}

El cultivo de L. vannamei se desarrolló por un período de 120 días en una empresa langostinera, ubicada en el departamento de Tumbes. Se utilizaron cuatro estanques de tierra de 4 hectáreas cada uno. El diseño del estudio correspondió a una investigación experimental clásica con dos réplicas: Estanques E1 y E2 como grupo control y el grupo EM conformado por los estanques E3 y E4.

\section{Acondicionamiento de estanques y siembra}

El agua para el cultivo fue obtenida durante las mareas altas desde un estero, dos veces por día, con una electrobomba centrifuga de 15 HP. Previamente a este proceso, se instaló una malla mosquitera $(0,8 \mathrm{~mm}$ de luz de malla) recubierta con malla anchovetera en el tubo de ingreso y en la salida de los estanques. Se sembraron post larvas (PLs) de $0.02 \mathrm{~g}$ aproximadamente, a una densidad de $15 \mathrm{PLs} /$ $\mathrm{m}^{2}$ (600 000 individuos por estanque) procedentes del laboratorio PROMARISCO, Ecuador, certificadas como SPF (Specific-pathogen-free) para el virus de la mancha blanca. Las PLs fueron transportadas en bolsas de polietileno suministradas con oxígeno. Antes de realizar la siembra en los estanques, las PLs fueron aclimatadas por 20 minutos, colocando las bolsas de polietileno en contacto con el agua del estanque.

\section{Preparación del probiótico}

Se empleó el probiótico comercial EM·CAMARÓN $\mathrm{TP}^{\circledR}$ (Bioem, Japón). Antes de la aplicación al estanque y alimento, se realizó el proceso de activación (EMA) y extendido(EME) del producto, añadiendo melaza como substrato para los microorganismos. Para la obtención de EMA y EME, se siguieron las recomendaciones del proveedor: EMA = EM·CAMARÓN TP $®(2$ litros $)+$ melaza (20 kg) + agua (1000 litros de volumen final);
$\mathrm{EME}=\mathrm{EMA}(50$ litros $)+\mathrm{EM} \cdot \mathrm{CAMARÓN} \mathrm{TP} \AA$ (0.5 litros.) + melaza (20 kg) + agua (1000 litros de volumen final) (Effective Microorganisms Research Organization, 2008).

\section{Manejo de la alimentación y aplicación del probiótico}

Se aplicó 425 litros de EME por hectárea al agua de los estanques semanalmente (E3 y E4). El alimento fue mezclado con tres litros de EMA adicionando tres litros de agua (dosis recomendada en la ficha técnica del probiótico comercial) por cada $100 \mathrm{~kg}$ para lograr una mejor homogenización. Este proceso se realizó, un día previo al suministro del alimento, empleando una mezcladora mecánica SUNCOO 4/5HP (EE.UU.) (Effective Microorganisms Research Organization, 2008).

La alimentación de los organismos se realizó a partir del día 17 después de la siembra, período en el cual las PLs subsisten con el alimento natural presente en el agua de los estanques. Se utilizó alimento comercial Nicovita al $35 \%$ de proteína, hasta que los individuos alcanzaron un peso de $5 \mathrm{~g}$ en promedio; a partir del cual se redujo el porcentaje de proteína del alimento comercial al $28 \%$ hasta el término del experimento. Las dosis alimentarias se establecieron en función de la edad (días de cultivo) y peso de los organismos de acuerdo con las tablas de alimentación de la empresa, $\mathrm{y}$, además, teniendo en cuenta las observaciones diarias del comportamiento y estado de salud de los individuos.

\section{Monitoreo del crecimiento, parámetros físico- químicos y materia orgánica}

El parámetro de crecimiento en peso fue monitoreado semanalmente. Hasta la tercera semana después de la siembra, la muestra se obtuvo con un chayo de malla fina. El peso, en esta fase, fue determinado con una probeta de $100 \mathrm{ml}$ aplicando el método volumétrico. A partir de la cuarta semana se capturó ejemplares al azar en cinco puntos de cada estanque y se utilizó una balanza electrónica de 0,01 $\mathrm{g}$ de sensibilidad (BL305S, Shimadzu - Japón). En ambos casos, 200 individuos de cada estanque fueron capturados. Se determinó el factor de conversión alimenticia $(\mathrm{FCA})=$ total alimento seco /ganancia de peso húmedo y la supervivencia $(\%)=$ Número final de langostinos/Número inicial de langostinos) x 100, al final del cultivo. 
La temperatura y oxígeno disuelto del agua de cultivo fueron monitoreados con un equipo multiparámetros YSI 550 (EE.UU.) diariamente. La salinidad, transparencia de agua, y $\mathrm{pH}$, fueron medidos semanalmente con un refractómetro Vital Sine SR6 (Japón), disco de Secchi y un potenciómetro WpH600 (China), respectivamente. Con la misma frecuencia se determinó el amonio y nitritos con un test de colorimetría marca Nutrafin (Aquaplus, China). Los parámetros de oxígeno $(\mathrm{mg} / \mathrm{l})$, temperatura $\left({ }^{\circ} \mathrm{C}\right)$, salinidad (ups), $\mathrm{pH}$, amonio y nitritos (mg/l) al inicio del cultivo fueron 4,96, 28,7, 18,3, 7,75, 0,31 y 0,29 para los estanques control, y 4,80, 27,5, 18,0, 7,62, 0,30 y 0,28 para los estanques con EM, respectivamente. La materia orgánica $(\mathrm{MO} \%)$ del suelo de los estanques fue analizada al inicio y término del cultivo, siguiendo un método previamente descrito (Kristensen et al., 1995). El porcentaje de MO se determinó en el laboratorio BIODES, Tumbes.

\section{Análisis de datos}

El parámetro de crecimiento en peso fue evaluado mediante análisis de varianza para un modelo encajado. Posteriormente, se obtuvo el peso promedio entre las repeticiones de cada grupo y se aplicó el análisis de varianza para un modelo factorial de dos factores fijos. $Y i j k=U+A i+B j+(A B) i j+E i j k$

Dónde:

Yijk : una medición cualquiera.

U : peso medio verdadero.

Ai : efecto del factor microorganismos eficaces sobre el crecimiento.
Bj : efecto del factor tiempo sobre el crecimiento. (AB)ij : efecto de la interacción de los dos factores sobre el crecimiento.

Eijk : error experimental.

La prueba de Tukey fue empleada para determinar a favor de qué grupo se presentaron las diferencias significativas en el crecimiento a través del tiempo. Los datos y análisis estadísticos fueron procesados con un nivel de significancia del 0,05 con el programa Microsoft Excel 2013.

Se determinó las producciones totales $=\mathrm{kg}$ de langostino cosechados por estanque, $\mathrm{y}$ bruta $=\mathrm{kg}$ de langostino cosechados por hectárea. La evaluación económica se realizó en base a los datos financieros del cultivo, proporcionados por la empresa. Los datos fueron determinados en dólares y luego extrapolados a la hectárea para obtener los siguientes indicadores: Ingresos netos $(\mathrm{IN})=$ Ingreso bruto - total de egresos; relación beneficio - costo $(\mathrm{RBC})=$ ingreso bruto / costo total del cultivo; merito económico $(\mathrm{ME})=$ costo total de alimento / ganancia en peso; retorno por dólar invertido $(\mathrm{RDI})=$ ingreso neto / costo del alimento.

\section{RESULTADOS}

\section{Crecimiento en peso}

La figura 1 muestra los pesos promedio de los langostinos, luego de 120 días de cultivo, donde se observa que el crecimiento de los individuos tratados con EM fue superior a los del control (sin EM).

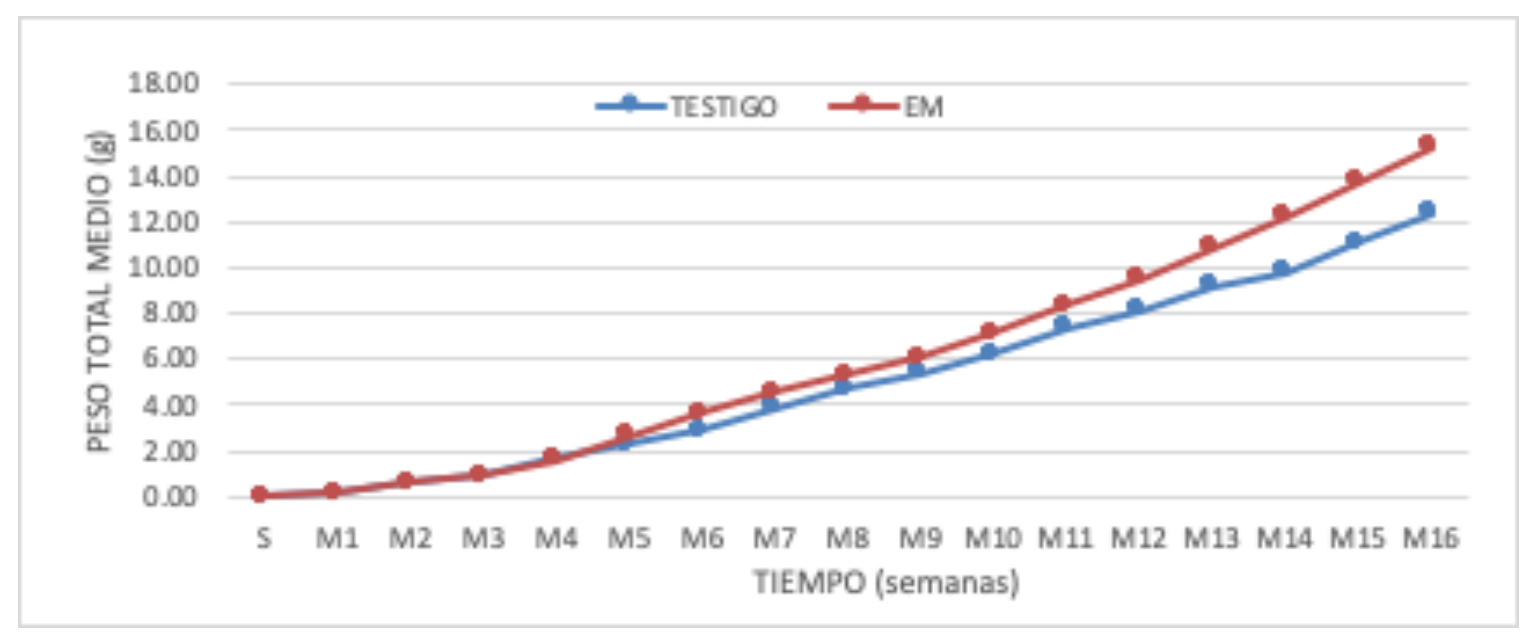

Figura 1. Pesos promedio semanales de L. vannamei, cultivados en sistema semi- intensivo, con suministro de microorganismos eficaces. S: peso medio de siembra, M: muestreo semanal, E: estanque. S: siembra, M: muestreo semanal. 
El análisis de varianza de los pesos demostró diferencias significativas entre el control y el grupo EM $(p=0,004)$ al término del experimento, encontrándose que los langostinos del grupo EM obtuvieron un mayor crecimiento $(15,25 \pm 1,24 \mathrm{~g})$ con respecto a los individuos del grupo control $(12,35 \pm 1,42 \mathrm{~g})$.

Se aplicó un análisis de varianza para determinar el efecto de los microorganismos eficaces $(\mathrm{p}=0,002)$, el tiempo $(\mathrm{p}=0,012)$ y la interacción de ambos $(\mathrm{p}=0,024)$ en el crecimiento en peso de los organismos cultivados, evidenciándose que existe efecto significativo de tales factores. La prueba de Tukey para comparar los pesos medios de los langostinos entre ambos grupos, mostró diferencias significativas en el crecimiento (peso) a favor del grupo EM a partir de la quinta semana de cultivo (tabla 1).

\section{Sobrevivencia, factor de conversión alimenticia (FCA) y producción}

La sobrevivencia en ambos grupos estuvo por debajo del $50 \%$. Los valores promedios de las réplicas evidenciaron que el control $(45,48 \%)$ superó ligeramente al tratamiento con EM (44,39\%). En cuanto a los FCA, se obtuvieron valores similares entre repeticiones del control $(1,59 \pm 0,20$ y $1,54 \pm 0,19)$ y del tratamiento con EM $(1,11 \pm 0,31$ y $1,23 \pm 0,29)$; sin embargo, el grupo EM presentó los mejores valores de este parámetro, con un promedio de $1,17 \pm 0,32$. Con respecto al rendimiento, las producciones brutas $(\mathrm{kg} /$ ha) fueron superiores en los estanques suministrados con EM: E3 y E4 con 1 001,28 y $1028,90 \mathrm{~kg} / \mathrm{ha}$, respectivamente (figura 2).

\section{Parámetros físico-químicos y materia orgánica}

Los niveles de oxígenos disuelto mostraron diferencias estadísticas $(p<0.05)$, obteniéndose mejores promedios en los estanques del grupo EM. Los valores obtenidos para la temperatura del agua no evidenciaron diferencias significativas, no obstante, la salinidad y transparencia fueron estadísticamente diferentes entre los estanques del grupo control y EM $(p<0.05)$. No hubo diferencias significativas en los valores de $\mathrm{pH}$, amonio no ionizado (NH3), nitritos (NO2) y materia orgánica, sin embargo, se observó una clara disminución de los niveles de los últimos tres parámetros en los estanques del grupo EM (tabla 2).

Tabla 1. Prueba de Tukey para determinar diferencias significativas entre los pesos medios de L. vannamei, cultivados en sistema semi-intensivo con suministro de microorganismos eficaces.

\begin{tabular}{ccccc}
\hline \multicolumn{5}{c}{ PESOS MEDIOS } \\
\hline TIEMPO & Control & EM & Diferencia & DMS \\
\hline M1 & 0,18 & 0,17 & 0,01 & 0,163 \\
M2 & 0,59 & 0,61 & 0,01 & 0,163 \\
M3 & 0,95 & 0,90 & 0,05 & 0,163 \\
M4 & 1,67 & 1,64 & 0,03 & 0,163 \\
M5 & 2,27 & 2,65 & $0,39^{*}$ & 0,163 \\
M6 & 2,91 & 3,65 & $0,74^{*}$ & 0,163 \\
M7 & 3,92 & 4,56 & $0,63^{*}$ & 0,163 \\
M8 & 4,71 & 5,30 & $0,60^{*}$ & 0,163 \\
M9 & 5,38 & 6,07 & $0,70^{*}$ & 0,163 \\
M10 & 6,26 & 7,17 & $0,91^{*}$ & 0,163 \\
M11 & 7,38 & 8,33 & $0,95^{*}$ & 0,195 \\
M12 & 8,11 & 9,51 & $1,40^{*}$ & 0,214 \\
M13 & 9,21 & 10,88 & $1,68^{*}$ & 0,214 \\
M14 & 9,83 & 12,25 & $2,42^{*}$ & 0,214 \\
M15 & 11,10 & 13,75 & $2,65^{*}$ & 0,214 \\
M16 & 12,35 & 15,25 & $2,91^{*}$ & 0,195 \\
\hline M: Muestreo semanal, DMS: Diferencia Mínima Significativa, *: valor significativo al $0.05 \%$
\end{tabular}




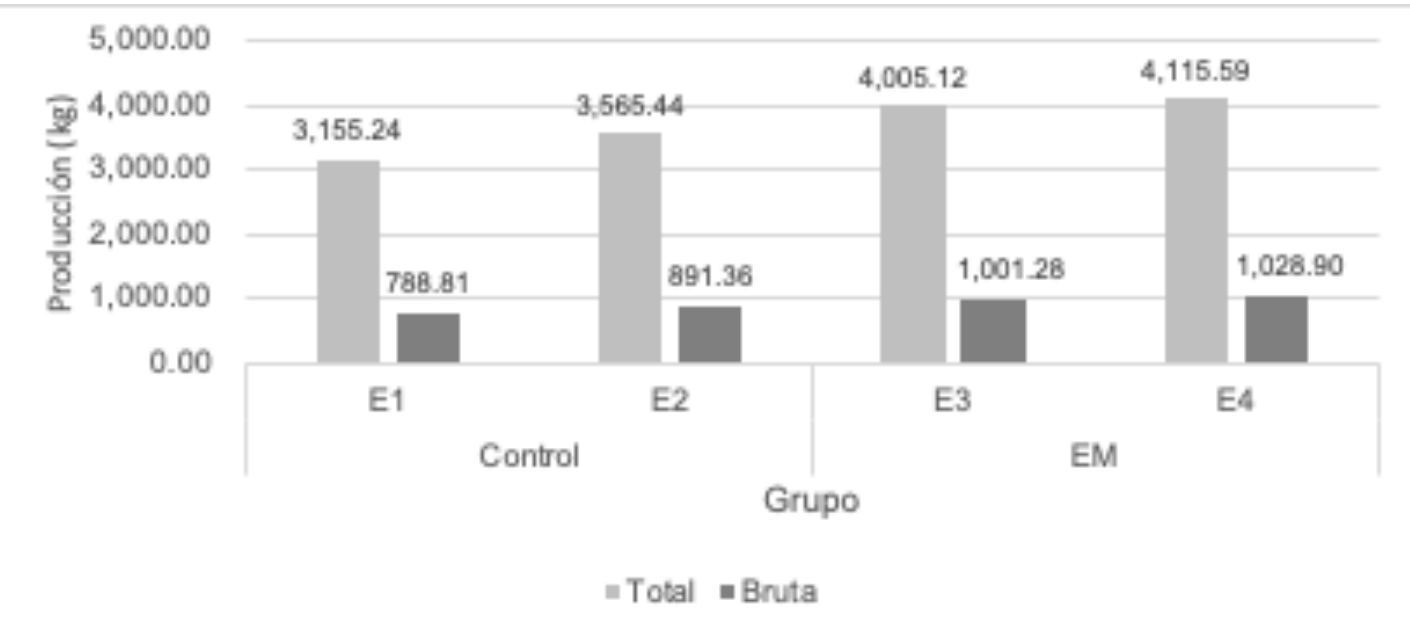

Figura 2. Producciones total y bruta del cultivo semi-intensivo de L. vannamei con suministro de microorganismos eficaces. E: estanque.

Tabla 2. Parámetros fisicoquímicos del agua y materia orgánica del cultivo de L. vannamei con suministro de microorganismos eficaces.

\begin{tabular}{|c|c|c|c|c|c|}
\hline \multirow{2}{*}{ Parámetro } & \multicolumn{2}{|c|}{ Control } & \multicolumn{2}{|c|}{ EM } & \multirow{2}{*}{ Valores óptimos } \\
\hline & E1 & E2 & $\mathbf{E 3}$ & E4 & \\
\hline \multicolumn{6}{|l|}{ Agua } \\
\hline Oxígeno (mg/l) & $4.86 \pm 1.45^{\mathrm{a}}$ & $5.15 \pm 1.32^{\mathrm{a}}$ & $6.31 \pm 2.04^{b}$ & $6.09 \pm 1.92^{\mathrm{b}}$ & $\begin{array}{c}4-6 \\
\text { (Chanratchakool et al., 2000) }\end{array}$ \\
\hline Temperatura $\left({ }^{\circ} \mathrm{C}\right)$ & $28.7 \pm 1.57^{\mathrm{a}}$ & $29.9 \pm 1.24^{\mathrm{a}}$ & $29.2 \pm 1.32^{\mathrm{a}}$ & $28.3 \pm 2.02^{\mathrm{a}}$ & $\begin{array}{c}25-32 \\
\text { (Boyd, 2001) }\end{array}$ \\
\hline Salinidad (ups) & $17.2 \pm 2.34^{\mathrm{a}}$ & $15.4 \pm 2.18^{\mathrm{a}}$ & $12.4 \pm 2.04^{b}$ & $13.7 \pm 2.31^{\mathrm{b}}$ & $\begin{array}{c}5-30 \\
\text { (Zhou et al., 2009) }\end{array}$ \\
\hline Transparencia $(\mathrm{cm})$ & $29.2 \pm 4.34^{\mathrm{a}}$ & $38.2 \pm 3.15^{\mathrm{b}}$ & $30.3 \pm 3.16^{\mathrm{a}}$ & $37.4 \pm 4.12^{\mathrm{b}}$ & $\begin{array}{c}15-35 \\
\text { (Zhou et al., 2009) }\end{array}$ \\
\hline $\mathrm{pH}$ & $7.98 \pm 0.42^{\mathrm{a}}$ & $8.01 \pm 0.12^{\mathrm{a}}$ & $7.08 \pm 0.32^{\mathrm{a}}$ & $6.98 \pm 0.37^{\mathrm{a}}$ & $\begin{array}{c}6.5-8.5 \\
\text { (Vinatea, 2001) }\end{array}$ \\
\hline NH3 (mg/l) & $0.33 \pm 0.23^{\mathrm{a}}$ & $0.30 \pm 0.15^{\mathrm{a}}$ & $0.10 \pm 0.33^{\mathrm{a}}$ & $0.21 \pm 0.16^{\mathrm{a}}$ & $\begin{array}{c}<0.3 \\
\text { (Lee y Wickins, 1992) }\end{array}$ \\
\hline NO2 (mg/l) & $0.29 \pm 0.35^{\mathrm{a}}$ & $0.39 \pm 0.33^{\mathrm{a}}$ & $0.20 \pm 0.28^{\mathrm{a}}$ & $0.19 \pm 0.43^{\mathrm{a}}$ & $\begin{array}{c}<0.25 \\
\text { (Lee y Wickins, 1992) }\end{array}$ \\
\hline \multicolumn{6}{|l|}{ Sedimento } \\
\hline Materia orgánica $(\%)^{*}$ & $6.20 \pm 0.73^{\mathrm{a}}$ & $5.97 \pm 0.61^{\mathrm{a}}$ & $5.20 \pm 0.25^{\mathrm{a}}$ & $4.98 \pm 0.67^{\mathrm{a}}$ & $\begin{array}{c}3-4 \\
\text { (Aguilar et al., 2009) }\end{array}$ \\
\hline
\end{tabular}

Superíndices diferentes en la misma fila muestran diferencia significativa $(\mathrm{p}<0.05)$. E: estanque. *: al término del cultivo.

\section{Evaluación económica}

Los diferentes indicadores económicos fueron numéricamente superiores en el tratamiento con EM. Entre estos, se enfatiza un mejor retorno por dólar invertido, indicador de interés para la acuicultura (tabla 3).

\section{DISCUSIÓN}

Existe sustento científico que demuestra que la mejor forma de usar los probióticos es en el alimento con el objetivo de enriquecer el tracto digestivo a través de la colonización y multiplicación de los microorganismos incorporados en la dieta, restando importancia a la aplicación de estos productos en el 
Tabla 3. Evaluación económica del cultivo semi-intensivo de L. vannamei, con suministro de microorganismos eficaces.

\begin{tabular}{lllll}
\hline Indicador & Ingreso neto $\mathbf{( \$ / h a )}$ & Relación beneficio-costo & $\begin{array}{l}\text { Mérito } \\
\text { económico (\$) }\end{array}$ & $\begin{array}{l}\text { Retorno por dólar } \\
\text { invertido (\$) }\end{array}$ \\
\hline Control & 1727.11 & 1.68 & 1.72 & 1.20 \\
EM & 3609.00 & 2.37 & 1.29 & 2.76 \\
\hline
\end{tabular}

Ha: hectárea, \$: dólar.

87, 349-353

medio acuático (Kumar et al., 2008; Irianto y Austin, 2002). Sin embargo, de acuerdo a los resultados del presente trabajo, se encontró diferencias significativas en el crecimiento de los organismos a favor del grupo suministrado con EM en agua y alimento. Esto se sustenta en que los microorganismos primero deben superar procesos de adaptación, establecimiento $\mathrm{y}$ colonización, lo cual puede tomar tiempo una vez que los probióticos son suministrados en los cultivos de especies acuícolas (Aguirre et al., 2012).

Otros estudios, en los cuales solo se utilizó probióticos constituidos por un solo grupo de microrganismos (bacterias acido lácticas o $S$. cerevisiae), reportaron pesos finales bajos con respecto a los de este estudio (Granillo, 2007; Sanjinez, 2006). Si bien en ambos casos se aplicó en el agua y alimento, los resultados se deberían probablemente a la baja dosis que utilizaron y al conglomerado de cepas que conforman cada probiótico, en donde la acción sinérgica de un conjunto de microorganismos, como las que contiene la tecnología EM, podría generar mejores efectos. Sin embargo, también podría atribuirse a la mayor sobrevivencia que obtuvieron (70\%), lo cual conllevó a tener más organismos en el estanque y por lo tanto menos espacio, limitando el crecimiento de los individuos.

Según estudios realizados en otros países, utilizando EM y otros probióticos conteniendo bacterias lácticas, se logró incrementar las sobrevivencias en el cultivo de camarón tigre Penaeus monodon (Alavandi et al., 2004; McIntosh et al., 2000). También se ha mejorado la sobrevivencia de Macrobrachium rosenbergii (camarón de río) adicionando cepas de Streptococcus cremoris, L. acidophilus, y L. bulgaricus directamente al agua (Ismail y Solima, 2010). A pesar de estos hallazgos, los resultados de sobrevivencia obtenidos en este trabajo (en ambos tratamientos) son contradictorios, dado que no se logró mejorar este parámetro. Una posible causa sería la influencia de los niveles fluctuantes de temperaturas y salinidad registradas, dado que estos parámetros son críticos en las respuestas fisiológicas de langostinos, que consecuentemente afecta la sobrevivencia (Valdez et al., 2008). Aunque, el control de estas variables se encuentra mayormente asociado a los cambios ambientales (precipitaciones) y al manejo operacional del cultivo (Cuéllar et al., 2010). Además, los niveles altos de MO (mala calidad del sedimento) podrían haber jugado un papel adverso sobre este parámetro, ya que en varios estudios se encontraron sobrevivencias superiores a $70 \%$, con niveles de $\mathrm{MO}$ inferiores a $2 \%$ (Novillo, 2005; Sanjinez, 2006; Granillo, 2007).

El alimento balanceado representa el 60-65 \% de los gastos de producción de L. vannamei (FAO, 2009). En este sentido, algunas experiencias de cultivo, en las cuales se utilizó alimento de alto porcentaje proteico (35\%) durante todo el cultivo, sin aplicación de probióticos, obtuvieron altos FCA (Granda y Puell, 2007; Vega, 2008). Sin embargo, en esta investigación, los FCA, fueron mejores en los estanques con suministro de EM. Esto debido probablemente al efecto de los microorganismos probióticos que secretan enzimas, las cuales ejercen un papel importante en los procesos de absorción y asimilación de los nutrientes del alimento (Balcázar et al., 2006; Alavandi et al., 2004).

En cuanto a las producciones brutas, estas fueron superiores en el grupo EM. Este hecho guarda relación con el mayor peso promedio obtenido en los estanques influenciados por los EM que ejercen un efecto benéfico tanto en el agua, suelo, alimento y microbiota de los individuos cultivados (Mahious y Ollevier, 2005). Además, la microbiota intestinal de los animales acuáticos es un reflejo de la diversidad de las comunidades microbianas que se encuentran en el agua, por lo que también es esencial mantener o promover la proliferación de microorganismos benéficos en el medio acuático para mejorar la producción de langostino (Ortega y Encalada, 2003; Balcázar et al., 2006).

En el presente estudio se observó que, en general, los parámetros fisicoquímicos del agua se mantuvieron dentro de los niveles adecuados 
para el cultivo de L. vannamei en un sistema semiintensivo (Zhou et al., 2009; Chanratchakool et al., 2000). Sin embargo, es importante resaltar que se registró diferencias significativas en cuanto a las concentraciones de oxígeno, con mejores niveles en el grupo con suministro de EM, lo cual se debería a la descomposición por procesos fermentativos, fundamentalmente anaeróbicos, de la materia orgánica ejercidos por los EM (Aguilar et al., 2009). Por otro lado, las variaciones en los parámetros de temperatura, salinidad y transparencia entre los tratamientos se debieron probablemente a aspectos propios de la naturaleza, los cuales no pueden ser controlados por los operarios, como intensidad solar extremas y fuertes precipitaciones observadas al inicio y hacia el final del estudio, tal como lo observado por Ramírez et al., (2006) para el camarón rosado Farfantepenaeus duorarum.

En cuanto al $\mathrm{pH}$, valores inferiores a 7 y superiores a 8 pueden afectar la circulación de la hemolinfa en los langostinos y aumentar las concentraciones de amonio a niveles tóxicos (Pretto, 1999; Hsien y Aguillón, 2008; Vinatea, 2001). En el presente trabajo, este parámetro estuvo dentro de esos límites. Si bien no hubo diferencias significativas entre los grupos, los valores fueron inferiores en el tratamiento con EM. Este hallazgo concuerda con los de otros estudios, donde los valores del $\mathrm{pH}$ fueron regulados al añadir bacterias ácido lácticas, las cuales mejoraron la calidad del agua, atribuyendo tal hecho a la capacidad de los microorganismos probióticos de generar compuestos orgánicos extracelulares que disminuyen el pH (Farzanfar, 2006; Qi et al., 2009; Rengpipat et al., 2008).

El uso de probióticos en otros trabajos muestra escaso o nulo efecto sobre la disminución de compuestos nitrogenados (Tejada et al., 2002; Ajitha et al., 2004). No obstante, en la presente investigación, las concentraciones de amonio no ionizado (NH3) y nitritos (NO2) mostraron una reducción en los estanques tratados con EM, pero no fueron diferentes estadísticamente con respecto al grupo control. Esta observación concuerda con otro trabajo en el que se utilizó microalgas perifíticas (cianobacterias y diatomeas) y Bacillus pumilus en el cultivo de $P$. monodon (Banerjee et al., 2010). Aspecto que se podría atribuir al hecho de que el nitrógeno y fósforo del alimento no consumido y de las heces son reciclados y procesados por microorganismos, reduciendo la disponibilidad de tales compuestos en el ambiente de cultivo (Khatoon et al., 2007).
Por otro lado, el porcentaje de MO en los estanques con suministro de EM mostró una reducción al término del estudio. En este caso la influencia de los EM pudo haber sido importante ya que las bacterias probióticas degradan la $\mathrm{MO}$ que suele acumularse en el sedimento de los estanques, debido a que estos compuestos orgánicos son metabolizados y transformados en biomasa celular, $\mathrm{C}_{2}$ y minerales (Kumar et al., 2008; Samocha, et al., 2004; Torres, 2005). Sin embargo, los valores obtenidos aún se consideran altos para la producción de animales acuáticos como el langostino, dado que la $\mathrm{MO}$ debe estar entre 3-5\% (Aguilar et al., 2009), lo cual podría atribuirse a un pobre manejo de calidad de agua en ciclos de cultivo anteriores a la intervención de este proyecto con el uso de EM, lo que permitió una acumulación en exceso de $\mathrm{MO}$ a través del tiempo. Por lo tanto, la aplicación de EM en un solo ciclo (4 meses) no sería suficiente para llevar tales valores a niveles aceptables para la especie, dado que los microorganismos probióticos necesitan adaptarse al medio, lo cual puede tomar tiempo (Aguirre et al., 2012).

El camarón blanco L. vannamei es una especie de alto valor económico, fundamentalmente en el mercado internacional como EE.UU. y Europa (Barón y Bückle, 2004; Valdez et al., 2008). El uso de EM representó solo el $2.16 \%$ de los costos de producción en los estanques E3 y E4 (incluyendo mano de obra extra) bajo las condiciones del presente estudio. Teniendo en cuenta este aspecto, la evaluación económica demostraría la viabilidad y rentabilidad del cultivo en ambos grupos, control y EM. No obstante, la producción de L. vannamei en el grupo EM, aun con la inversión adicional, conllevó a la obtención de mejores indicadores económicos. Esto estaría relacionado con la forma de comercialización en el mercado internacional bajo la modalidad de entero, debido al mayor peso promedio obtenido lo que incrementó el precio por kilogramo de los langostinos de este grupo.

Dado que los resultados no mejoraron otros parámetros como la sobrevivencia, se considera que se podrían mejorar junto a otras variables con el uso continuo de EM para lograr el establecimiento de comunidades microbianas benéficas. Además, es necesario seguir investigando diferentes niveles de EM para determinar dosis óptimas para esta especie, así como realizar monitoreos microbiológicos para evaluar las variaciones de las poblaciones bacterianas y fúngicas benéficas en el agua como en los organismos cultivados. 


\section{CONCLUSIONES}

El uso de EM mejoró el crecimiento en peso de los langostinos en 120 días de cultivo; asimismo, generó mayores ingresos netos, una mejor relación beneficio costo y merito económico, por lo que se recomendaría su inclusión en la producción semi-intensiva del langostino.

Los estanques tratados con EM mostraron mejores parámetros físico-químicos del agua para el cultivo de esta especie, incluyendo un porcentaje menor de materia orgánica en el fondo de los estanques, sugiriendo una reducción en la contaminación de los cuerpos de agua naturales ocasionados por los efluentes de esta actividad.

\section{Correspondencia}

Marcos Enrique Serrano Martínez

Correo electrónico: enrique.serrano@upch.pe

\section{REFERENCIAS BIBLIOGRAFICAS}

1. Aguilar, F., Shintani, M., \& Tabora, P. (2009). Aplicación de Microorganismos Eficaces (EM) en producción agropecuaria y manejo de desechos. Universidad EARTH.

2. Aguirre, G., Lara, M., Sánchez, J., Campa, A., \& Luna, A. (2012). Una revisión sobre el uso de probióticos en el cultivo de organismos acuáticos. Aquacultura, 98,22-26.

3. Ajitha, S., Sridhar, M., Sridhar, N., Singh, I., \& Varghese, V. (2004). Probiotic Effects of Lactic Acid Bacteria against Vibrio alginolyticus in Penaeus indicus (H. Milne Edwards). Asian Fisheries Science, 17, 71-80.

4. Alavandi, V., Vijayan, K., Santiago T., Poornima, M., Jithendran, K., Ali, S., \& Rajan, J. (2004). Evaluation of Pseudomonas sp. PM11 and Vibrio fluvialis PM17 on immune indices of tiger shrimp, Penaeus monodon. Fish Shellfish Immunol, 17, 115-120.

5. Balcázar, L., Blas, I., Ruiz, I., Cunningham, D., Vendrell, D., \& Múzquiz, J. (2006). The role of probiotics in aquaculture. Veterinary Microbiology, 114, 173-186.

6. Banerjee, S., Khatoon, H., Shariff, M., \& Yusoff, F. (2010). Enhancement of Penaeus monodon shrimp post larvae growth and survival without water exchange using marine Bacillus pumilus and periphytic microalgae. Fisheries Science, 76, 481487.

7. Barón, B., \& Bückle, L. (2004). Intensive culture of Litopenaeus vannamei BOONE 1931, in a recirculating seawater system. Ciencias Marinas, 30,
179-188.

8. Boyd, C. E. (2001). Consideraciones sobre la calidad del agua y del suelo en cultivos de camarón. En Haws, M. C., Boyd, C.E. (eds.). Métodos para Mejorar la Camaronicultura en Centroamérica. (pp 24-25). Editorial Imprenta UCA.

9. Campa, A., Luna, A., Mazón, J., Aguirre, G., Ascencio, F., \& González, H. (2011). Efecto de bacterias probióticas en el cultivo larvario del ostión de placer Crassostrea corteziensis (Bivalvia: Ostreidae). Revista de Biología Tropical, 59, 183191.

10. Chae, M., Yun-Seok, C., \& Kye-Heon, O. (2009). Removal of pathogenic bacteria and nitrogens by Lactobacillus spp. JK-8 and JK-11. Aquaculture, 287, 266-270.

11. Chanratchakool, P., Fegan, D.F. \& Phillips, M.J.(2020). Thailand. In: Thematic review on Management Strategies for Major Diseases in Shrimp Aquaculture. A component of the WB/NACA/WWF/ FAO Program on Shrimp Farming and the Environment. Report of the workshop held in Cebu, Philippines 1999. (pp.85-90).

12. Cuéllar, J., Lara, C., Morales V, De Gracia, A., \& García, O. (2010). Manual de buenas prácticas de manejo para el cultivo del camarón blanco Penaeus vannamei. OIRSA-OSPESCA.

13. Effective Microorganisms Research Organization. (2008). Producción animal. Tecnología EM ${ }^{\mathrm{TM}}$. https:// www.em-la.com/aplicacoes-e-uso/producao-animal/

14. FAO (2009). Impact of rising feed ingredient prices on aquafeeds and aquaculture production. FAO Fisheries and Aquaculture Technical Paper. No. 541. Rome. 63p.

15. Farzanfar, A. (2006). The use of probiotics in shrimp aquaculture. FEMS Immunology \& Medical Microbiology, 48, 149-158.

16. Granda, M., \& Puell, L. (2007). Producción de Penaeus vannamei en cultivo semi-intensivo con tres marcas de alimento balanceado en langostinera Mar Norte S.A. [Tesis para optar el título de Ingeniero Pesquero] Universidad Nacional de Tumbes.

17. Granillo, G. (2007). Incidencia en el Incremento de Peso y Consumo de Alimento de Litopenaeus vannamei con tres Tratamientos de Probióticos en el alimento y en el medio. [Tesis para optar el título de Ingeniero Acuícola]. Universidad Técnica de Machala.

18. Guillén, R. (2005). Relación de Bacterias Totales con Microorganismo Patógenos (Vibrios - Pseudomonas) en el Cultivo de Camarón. [Tesis para optar el título de Ingeniero Acuícola]. Universidad Técnica de Machala.

19. Günther, J., \& Montealegre, R. (2004). Efecto del probiótico Bacillus subtilis sobre el crecimiento y alimentación de tilapia (Oreochromis niloticus) y 
langostino (Macrobrachium rosenbergii) en laboratorio. Revista de Biología Tropical, 52, 937943.

20. Hendrickx, E. (1996). Los camarones Penaoidea bentónicos (Crustacea: Decápoda: Dendrobranchiata) del Pacifico mexicano. Comisión Nacional para el Conocimiento y la Biodiversidad CONABIO. Instituto de Ciencias del Mar y Limnología.

21. Higa, T., \& Parr, J. (1994). Beneficial and effective microorganisms for a sustainable agriculture and environment. International Nature Farming Research Center.

22. Hsien, S., \& Aguillón, C. (2008). Manual sobre "Reproducción y cultivo del camarón blanco (Litopenaeus vannamei). Cendepesca.

23. Irianto, A., \& Austin, B. (2002). Probiotics in aquaculture. Journal of Fish Diseases, 25, 633-642.

24. Ismail, M., \& Soliman, W. (2010). Studies on Probiotic Effects of Lactic Acid Bacteria against Vibrio vulnificus in freshwater Prawn Macrobrachium rosenbergii. American Journal of Science, 6, 781787.

25. Jiang, D., Lawrence, L., Neill, W., \& Gong, H. (2000). Effects of temperature and salinity on nitrogenous excretion by Litopenaeus vannamei juveniles. Journal of Experimental Marine Biology and Ecology, 253, 193-209.

26. Lee, D., \& Wickins J. (1992). Crustacean farming: ranching and culture. Blackwell Sci Publ.

27. Kesarcodi, A., Kaspar, H., Lategan, M., \& Gibson, L. (2008). Probiotics in aquaculture: The need, principles and mechanisms of action and screening processes. Aquaculture, 274, 1-14.

28. Khatoon H, Yusoff FM, Banerjee S, Shariff M, Mohamed S (2007). Use of periphytic cyanobacterium and mixed diatoms coated substrate for improving water quality, survival and growth of Penaeus monodon post larvae. Aquaculture, 271, 196-205.

29. Kristensen, E., Ahmed, S., \& Deval, A. (1995). Aerobic and anaerobic decomposition of organic matter in marine sediment: which is fastest? Limnology Oceanography, 40, 430-437.

30. Kumar, M., Swarnakumar, N., Sivakumar, K., Thangaradjou, T., \& Kannan, L. (2008). Probiotics in aquaculture: importance and future perspectives. Indian Journal of Microbiology, 48, 299-308.

31. Mahious, A., \& Ollevier, F. (2005). Probiotics and prebiotics in aquaculture. [Review]. 1st. Regional Workshop on techniques for enrichment on live food for used in larviculture. Urnia, Irán.

32. McIntosh, R., Samocha, T., Jones, E., Lawrence, A., McKee, D., Horowitz, S., \& Horowitz, A. (2000). The effect of a commercial bacterial supplement on the high-density culturing of Litopenaeus vannamei with a low-protein diet in an outdoor tank system and no water exchange. Aquacultural Engineering, 21,
215-227.

33. Nguyen, T., Ngoc, V., \& Merckx, R. (2012). Importancia del oxígeno disuelto en un sistema extensivo de cultivo de camarón en Vietnam. Revista de Aquacultura, 8, 54-64.

34. Novillo, G. (2005). Uso de Biorremediadores en Piscinas Camaroneras. [Tesis para optar el título de Ingeniero Acuícola]. Universidad Técnica de Machala.

35. Ortega, P., \& Encalada, J. (2003). Estudio de factibilidad de camarón (Penaeus vannamei) sostenible en Balao, Ecuador. [Tesis de licenciatura], Universidad Earth.

36. Paillard, C., Rox, F., \& Borrego, J. (2004). Bacterial disease in marine bivalves, a review of recent studies: Trends and evolution. Aquatic Living Resources , 17, 447-498.

37. Pretto, R. (1999). Manual de cría de camarones peneidos en estanque de aguas salobres. Editorial Guillermo Ríos Durán.

38. Pruzzo, C., Gallo, G., \& Canesi, L. (2005). Persistence of vibrios in marine bivalves: the role of interactions with haemolymph components. Environmental Microbiology, 7, 761-772.

39. Qi, Z., Zhang, X., Boon, N., \& Bossier, P. (2009). Probiotic in aquaculture of China-Current state, problems and prospect. Aquaculture, 290, 15-21.

40. Ramírez, M., Arreguín, F., \& Lluch, D. (2006). Efecto de la temperatura superficial y la salinidad en el reclutamiento del camarón rosado Farfantepenaeus duorarum (Decapoda: Penaeidae), en la Sonda de Campeche, Golfo de México. Revista de Biología Tropical, 54, 1241-1245.

41. Rengpipat, S., Rueangruklikhit, T., \& Piyatiratitivorakul, S. (2008). Evaluations of lactic acid bacteria as probiotics for juvenile seabass Lates calcarifer. Aquaculture Research, 39, 134-143.

42. Samocha, T., López, M., Jones, I., Jackson E., \& Lawrence, A. (2004). Characterization of intake and effluent waters from intensive and semi-intensive shrimp farms in Texas. Aquaculture Research, 35, 321-339.

43. Sanjinez, E. (2006). Alimentación de Penaeus vannamei utilizando dos marcas de alimento balanceado comercial en un cultivo semi-intensivo en la empresa langostinera Domingo Rodas S.A. [Tesis para optar el título de Ingeniero Pesquero]. Universidad Nacional de Tumbes.

44. Tejada, J., Tabora, P., Okumoto, S., Shintani, M., Vaquedano, I., \& Roque C. (2002). Organic shrimp production: Opportunities with EM (Effective Microorganisms) applications. Universidad EARTH.

45. Torres, B. (2005). Organic matter decomposition in simulated aquaculture ponds. [PhD. Thesis]. Wageningen University.

46. Valdez, G., Díaz, F., Re, A., \& Sierra, E. (2008). Efecto 
de la salinidad sobre la fisiología energética del camarón blanco Litopenaeus vannamei (Boone). Hidrobiológica, 18, 105-115.

47. Vega, L. (2008). Manejo del alimento balanceado en el cultivo semi-intensivo de Penaeus vannamei en la empresa langostinera Domingo Rodas S.A. [Tesis para optar el título de Ingeniero Pesquero]. Universidad Nacional de Tumbes.
48. Villamil, L., \& Silva, M. (2009). Probióticos como herramienta biotecnológica en el cultivo de camarón: reseña. Boletín de Investigaciones Marinas $y$ Costeras, 38, 165-187.

49. Vinatea, L. (2001). Principios Químicos de Calidad de Agua en Acuicultura, una revisión para peces y camarones. Departamento de Acuicultura, Universidad Federal De Santa Catarina, Brasil.

50.Zhou, X., Wang, Y., \& Li, W. (2009) Effect of probiotic on larvae shrimp (Penaeus vannamei) based on water quality, survival rate and digestive enzyme activities. Aquaculture, 2,349-353. 